MATHEMATICS OF COMPUTATION

Volume 67, Number 221, January 1998, Pages 237-255

S $0025-5718(98) 00885-0$

\title{
A NEW CLASS OF ASYNCHRONOUS ITERATIVE ALGORITHMS WITH ORDER INTERVALS
}

\author{
J. C. MIELLOU, D. EL BAZ, AND P. SPITERI
}

\begin{abstract}
This paper deals with a new class of parallel asynchronous iterative algorithms for the solution of nonlinear systems of equations. The main feature of the new class of methods presented here is the possibility of flexible communication between processors. In particular partial updates can be exchanged. Approximation of the associated fixed point mapping is also considered. A detailed convergence study is presented. A connection with the Schwarz alternating method is made for the solution of nonlinear boundary value problems. Computational results on a shared memory multiprocessor IBM 3090 are briefly presented.
\end{abstract}

\section{INTRODUCTION}

In the past few years a large number of parallel computing methods have been proposed for the solution of large scale numerical problems (see Bertsekas and Tsitsiklis [7], Hockney and Jesshope [22], Ortega [30], and Schendel [34]). Among parallel algorithms asynchronous iterative methods have received a considerable amount of attention. Asynchronous algorithmic models were introduced by Chazan and Miranker (see [8]) for the solution of linear systems of equations. A necessary and sufficient condition of convergence is given in [8] (see also [2]).

In the nonlinear case, the convergence properties of parallel asynchronous iterative algorithms are now well understood. Many authors have concentrated on fixed point problems. In particular Miellou (see [24] and [26]) and Baudet (see [3]) have shown contraction properties using a vectorial norm (see also [33]). El Tarazi [15] has shown a contraction property using an appropriate scalar norm.

A complementary approach dealing with the properties of the nonlinear equations operator was considered by Miellou and Spiteri [29], Giraud Spiteri [20], and Spiteri [35] (see also [36]); the authors quoted above have proposed sufficient conditions of convergence for asynchronous iterations. The reader is referred to Giraud and Spiteri [21] for an implementation of asynchronous algorithms.

Some authors have also made use of the discrete maximum principle. Monotone sequences of vectors are generated in this case. Miellou [25] has given a sufficient condition of convergence for asynchronous iterations under partial ordering. Other contributions are due to El Tarazi [16], Miellou [27], El Baz (see [10], [12], and

Received by the editor December 9, 1994 and, in revised form, January 26, 1996 and July 19, 1996.

1991 Mathematics Subject Classification. Primary 65N12, 65N55, 68Q10, 68Q22.

Key words and phrases. Parallel iterative methods, asynchronous iterations, Schwarz alternating method, domain decomposition methods, boundary value problems.

(C)1998 American Mathematical Society 
[13]), Frommer (see [18] and [19]). Reference is also made to Cousot [9] for a study related to the proof of programs using fixed point techniques.

Finally, we note that Bertsekas has proposed a different approach based on the definition of a sequence of nested level sets (see [5] and [7], see also [4], [6], and [11]). Perturbation of fixed point iterative algorithms has been studied in this context by Miellou, Cortey-Dumont, and Boulbrachêne (see [28]).

In this paper we consider partial ordering techniques and propose new parallel asynchronous iterative methods which are more general than the ones quoted above. An original feature of the new parallel iterative methods presented here is flexible communication between processors. More precisely, updating phases can use data which are issued from computations in progress. This leads to a better coupling between communication and computation. In contrast with previous studies (see [3], [8], and [25]), the methods presented here permit one to consider block iterative schemes with delayed access to block subvectors, the components of which are relative to different iteration numbers or intermediate computations not explicitly labelled by an iteration number. Another important feature of this paper is the use of mappings which approximate the solution. A detailed convergence study is presented. The new model of parallel asynchronous iterations proposed in this paper can be applied with success to subdomain methods for the solution of partial differential equations and gradient type methods for network flow problems. Computational studies using shared memory and distributed memory multiprocessors are presented in detail in two complementary papers (see [14] and [37]). Computational experiences have shown the practical interest of the proposed parallel asynchronous iterative methods. A brief presentation of numerical results for the solution of partial differential equations via subdomain methods is made at the end of the paper.

The paper contains five sections. In Section 2 we present the main problem and give some background material. Section 3 deals with the new class of parallel asynchronous iterative methods. Two kinds of approximations of the fixed point mapping are presented in Section 4. In Section 5 we consider the application of the theoretical study to the Schwarz alternating method for the numerical solution of boundary value problems; computational experience using a shared memory multiprocessor IBM 3090 is also presented in this section.

\section{Problem statement}

2.1. Notations, background material. Let $n$ be a positive integer and assume that

$$
\begin{aligned}
& a \text { is a continuous mapping of } R^{n} \text { into } R^{n} \text {, } \\
& a \text { is a surjective } M \text {-function. }
\end{aligned}
$$

We recall that the mapping $a$ is an $M$-function (see [31] and [32]) if $a$ is off-diagonally antitone i.e. for any $x \in R^{n}$, the functions defined as follows:

$$
\left\{\begin{array}{l}
a_{l k}:\left\{t \in R \mid x+t e_{k} \in R^{n}\right\} \rightarrow R, \\
a_{l k}(t)=a_{l}\left(x+t e_{k}\right), l \neq k, l, k=1, \ldots, n,
\end{array}\right.
$$

are monotone decreasing, where $e_{k} \in R^{n} k=1, \ldots, n$, are the unit canonical basis vectors and furthermore $a$ is inverse isotone i.e.

$$
a(x) \leq a(y) \text { for any } x, y \in R^{n} \text { implies that } x \leq y .
$$


In the sequel we will make use of the following notation: for any sequence $\left\{x^{k}\right\} \subset$ $R^{n}, \lim _{k \rightarrow \infty} x^{k}=+\infty\left(\lim _{k \rightarrow \infty} x^{k}=-\infty\right)$ if $\lim _{k \rightarrow \infty} x_{i}^{k}=+\infty\left(\lim _{k \rightarrow \infty} x_{i}^{k}=\right.$ $-\infty)$ for at least one index $i$.

Definition 1. The mapping $a: R^{n} \rightarrow R^{n}$ is order-coercive if for any sequence $\left\{x^{k}\right\} \subset R^{n}$, such that $x^{k} \leq x^{k+1}, k=0,1, \ldots$, and $\lim _{k \rightarrow \infty} x^{k}=+\infty$, we have $\lim _{k \rightarrow \infty} a\left(x^{k}\right)=+\infty$ and for any sequence $\left\{x^{k}\right\} \subset R^{n}$, such that $x^{k} \geq x^{k+1}, k=$ $0,1, \ldots$, and $\lim _{k \rightarrow \infty} x^{k}=-\infty$, we have $\lim _{k \rightarrow \infty} a\left(x^{k}\right)=-\infty$.

We can characterize the surjectivity of $M$-functions by means of the ordercoercivity concept (see [32, Theorem 3.7]).

Theorem 1. Let $a: R^{n} \rightarrow R^{n}$ be a continuous $M$-function. Then a is surjective if and only if $a$ is order-coercive.

We consider now the solution of the following system of equations

$$
a(y)=0 .
$$

Under the above assumptions problem (2.5) has a unique solution.

2.2. Subproblem decomposition and associated fixed point mapping. Let $E=R^{n}$ and consider the following splitting of $E: E=\prod_{i=1}^{\alpha} E_{i}$, where $\alpha$ is a positive integer, $E_{i}=R^{n_{i}}$, and $\sum_{i=1}^{\alpha} n_{i}=n$. Each subspace $E_{i}$ is endowed with the natural (or componentwise) partial ordering associated with the cone $K_{i}=R_{+}^{n_{i}}$ of vectors with non-negative components in $R^{n_{i}}$. Let $w \in E$. Consider the following block decomposition of $w$ :

$$
w=\left\{w_{1}, \ldots, w_{i}, \ldots, w_{\alpha}\right\} \in \prod_{i=1}^{\alpha} E_{i},
$$

and the following block decomposition of $a$ :

$$
a(w)=\left\{a_{1}(w), \ldots, a_{i}(w), \ldots, a_{\alpha}(w)\right\} \in \prod_{i=1}^{\alpha} E_{i} .
$$

In the sequel $a_{i}\left(w_{1}, \ldots, w_{i-1}, y_{i}, w_{i+1}, \ldots, w_{\alpha}\right)$ will also be denoted by $a_{i}\left(y_{i} ; w\right)$. Let assumptions (2.1) and (2.2) hold. Then

$$
\left\{\begin{array}{l}
\text { for all } i \in\{1, \ldots, \alpha\} \text { and all } w \in E \text {, the mapping: } y_{i} \rightarrow a_{i}\left(y_{i}, w\right), \\
\text { is a continuous surjective } M \text {-function of } R^{n_{i}} \text { onto } R^{n_{i}}
\end{array}\right.
$$

(see [32, Theorem 3.5]). Moreover it follows from the assumption (2.2) that

$$
\left\{\begin{array}{l}
\text { for all } w \in E \text { and } i \in\{1, \ldots, \alpha\}, \text { the problem } a_{i}\left(z_{i} ; w\right)=0, \\
\text { has a unique solution } z_{i} .
\end{array}\right.
$$

Thus the mapping $F$ from $R^{n}$ onto $R^{n}$ such that

$$
F(w)=z=\left\{z_{1}, \ldots, z_{i}, \ldots, z_{\alpha}\right\},
$$

with $z_{i}$ from (2.7) is well-defined. It is a fixed point formulation for problem (2.5) since

$$
F(y)=y \Leftrightarrow a(y)=0 .
$$

Proposition 1. F is isotone on $E$. 
Proof. Let $w$ and $w^{\prime} \in E$ such that $w \leq w^{\prime}$. Let $z=F(w)$ and $z^{\prime}=F\left(w^{\prime}\right)$, where $z_{i}^{\prime}$ satisfies $a_{i}\left(z_{i}^{\prime} ; w^{\prime}\right)=0, i \in\{1, \ldots, \alpha\}$. By the off-diagonal antitonicity of $a$ we have

$$
a_{i}\left(z_{i}^{\prime} ; w\right) \geq 0, \quad i \in\{1, \ldots, \alpha\} .
$$

Thus for all $i \in\{1, \ldots, \alpha\}$, we have

$$
a_{i}\left(z_{i}^{\prime} ; w\right) \geq a_{i}\left(z_{i} ; w\right)=0,
$$

and by the inverse isotonicity of $a_{i}\left(z_{i} ; w\right)$, we have for all $i \in\{1, \ldots, \alpha\}$

$$
z_{i} \leq z_{i}^{\prime} \text {. }
$$

\subsection{Supersolutions and $a$-supersolutions.}

Definition 2. A vector $y \in E$ is a supersolution (a subsolution) if $F(y) \leq y$ $(F(y) \geq y)$.

Definition 3. A vector $y \in R_{+}^{n}$ is an $a$-supersolution (an $a$-subsolution) if $a(y) \geq 0$ $(a(y) \leq 0)$.

Proposition 2. If $y$ is an a-supersolution, then $y$ is a supersolution.

Proof. It follows from (2.7) and (2.8) that for all $i \in\{1, \ldots, \alpha\}$, we have

$$
a_{i}\left(F_{i}(y), y\right)=0 \leq a_{i}(y) .
$$

By the inverse isotonicity of $a_{i}$ we have

$$
F_{i}(y) \leq y_{i} .
$$

Remark 1. In general the converse is false. For example, consider the case where $a$ is a linear mapping. Let $A$ be the associated matrix, then it follows from assumption (2.2) that $A$ is an $M$-matrix. We have

$$
a_{i}(y)=\sum_{j=1}^{\alpha} A_{i j} y_{j}
$$

where $\left\{A_{i j}\right\}$ results from the block decomposition of $A$. The block diagonal matrices $A_{i i}$, are necessarily $M$-matrices. We have also

$$
y_{i}-F_{i}(y)=y_{i}+\sum_{j \neq i} A_{i i}^{-1} A_{i j} y_{j} .
$$

In particular, we note that $y_{i}-F_{i}(y)>0$, does not imply $A_{i i}\left(y_{i}-F_{i}(y)\right)=a_{i}(y)>0$. Indeed, consider the case where $E_{i}=R^{2}$ and $A_{i i}=\left|\begin{array}{cc}1 & -0,5 \\ -0,5 & 1\end{array}\right|$; then for $y_{i}-F_{i}(y)=$ $\left\{\frac{1}{10}, 1\right\}^{T}$, we find that

$$
A_{i i}\left(y_{i}-F_{i}(y)\right)=a_{i}(y)=\left\{-\frac{2}{5}, \frac{19}{20}\right\}^{T} .
$$

However in the linear case if $F$ corresponds to the Jacobi mapping associated with a point decomposition of the $M$-matrix $A$ (i.e. $\alpha=n$ and $E_{j}=R, j \in\{1, \ldots, n\}$ ), then $w \geq F(w)$ is equivalent to $A w \geq 0$.

Proposition 3. Let $a$ be an $M$-function and $w \in R^{n}$ an a-supersolution. Let $s \subset\{1, \ldots, n\}$ and $v \in R^{n}$, be such that $v \leq w, a_{i}\left(v_{i} ; w\right) \geq 0$, for all $i \in s$ and $v_{i}=w_{i}$, for all $i \notin s$, then $v$ is an a-supersolution. 
Proof. For all $i \in\{1, \ldots, n\}$ we have $a_{i}\left(v_{i} ; w\right) \geq 0$, since $w$ is an $a$-supersolution. Thus by the off-diagonal antitonicity of $a$ we find that

$$
a_{i}\left(v_{i} ; v\right) \geq a_{i}\left(v_{i} ; w\right) \geq 0 .
$$

Proposition 4. Let $a$ be an $M$-function, $v$ and $w$ two a-supersolutions. Then $v \wedge w=\left\{\min \left\{v_{1}, w_{1}\right\}, \ldots, \min \left\{v_{r}, w_{r}\right\}, \ldots, \min \left\{v_{n}, w_{n}\right\}\right\}$ is an a-supersolution.

Proof. We use the following notation: $a(v \wedge w)=\left\{a_{1}(v \wedge w), \ldots, a_{r}(v \wedge w), \ldots\right.$, $\left.a_{n}(v \wedge w)\right\}$, where $a_{r}$ corresponds here to a point decomposition of the mapping $a$. For all $r \in\{1, \ldots, n\}$, we have

$$
a_{r}(v \wedge w)=a_{r}\left(v_{r} \wedge w_{r} ; v \wedge w\right) .
$$

By the off-diagonal antitonicity of $a$, we have for all $r \in\{1, \ldots, n\}$,

$$
a_{r}\left(w_{r} ; v \wedge w\right) \geq a_{r}(w) \geq 0, \text { and } a_{r}\left(v_{r} ; v \wedge w\right) \geq a_{r}(v) \geq 0 .
$$

Thus, $a_{r}(v \wedge w) \geq 0$.

\section{Asynchronous iterations With ORDER INTERVALS}

In Section 2 we have considered a fixed point mapping associated with the exact solution of the subproblems (2.7). In this section we study fixed point mappings associated with approximate solutions. Such mappings are introduced for the solution of problem (2.5) via parallel asynchronous iterative methods with flexible communication.

3.1. Supermappings and $a$-supermappings associated with $F$. Throughout the paper we adopt the following notation for order intervals. Let $x_{i}, y_{i} \in E_{i}$, be such that $x_{i} \leq y_{i}$; then an order interval in $E_{i}$ is denoted by

$$
\left\langle x_{i}, y_{i}\right\rangle_{i}=\left\{z_{i} \in E_{i} \mid x_{i} \leq z_{i} \leq y_{i}\right\} .
$$

Similarly, let $x, y \in E$, be such that $x \leq y$; then an order interval in $E$ is denoted by

$$
\langle x, y\rangle=\{z \in E \mid x \leq z \leq y\} .
$$

Definition 4. Let $a$ be an $M$-function. Then the mapping $F^{a}$ with components $F_{i}^{a}$ is an $a$-supermapping associated with $F$ if for all $i \in\{1, \ldots, \alpha\}$ and $y$ elements of the domain of definition of $F_{i}^{a}:\left\{y \in E \mid a_{i}(y) \geq 0\right\}$, we have $F_{i}^{a}(y) \leq y_{i}$, $a_{i}\left(F_{i}^{a}(y), y\right) \geq 0$, and $F_{i}^{a}(y) \neq y_{i}$ if $F_{i}(y) \neq y_{i}$.

Definition 5. Let $a$ be an $M$-function. Then the mapping $F^{a}$ with components $F_{i}^{a}$ is a supermapping associated with $F$ if for all $i \in\{1, \ldots, \alpha\}$ and $y$ element of the domain of definition of $F_{i}^{a}:\left\{y \in E \mid a_{i}(y) \geq 0\right\}$, we have $F_{i}^{a}(y) \in\left\langle F_{i}(y), y_{i}\right\rangle_{i}$ and $F_{i}^{a}(y) \neq y_{i}$ if $F_{i}(y) \neq y_{i}$.

Proposition 5. If $F^{a}$ is an a-supermapping associated with $F$, then $F^{a}$ is a supermapping associated with $F$.

Proof. From (2.7), (2.8), and Definition 4 it follows that

$$
a_{i}\left(F_{i}(y) ; y\right)=0 \leq a_{i}\left(F_{i}^{a}(y) ; y\right) .
$$

Then

$$
F_{i}(y) \leq F_{i}^{a}(y),
$$

since $a_{i}$ is inverse isotone. 
Remark 2. The converse of Proposition 5 is in general false. This result can be shown using an argument similar to the one developed in Remark 1.

Remark 3 . We can analogously define submappings and $a$-submappings associated with $F$ by reversing the inequalities.

3.2. A new class of parallel asynchronous iterations. In this section we define a general class of iterative methods which contains in particular sequential relaxation methods like Jacobi or Gauss-Seidel and parallel iterative methods whereby components are updated simultaneously by concurrent processors without any order nor synchronization. The new class of asynchronous iterations presented here allows also flexible communication between processors. We introduce first the concepts of steering and sequence of delays.

Definition 6. A steering $\mathcal{S}$ of components of the iteration vector, is a sequence $\{s(p)\}$ where $p \in N$, the set of natural integers, such that

$$
\text { for all } p \in N, \quad s(p) \in\{1, \ldots, \alpha\} \text {. }
$$

Definition 7. A sequence $\{r(p)\}$ (or $\mathcal{R}$ ) of maximal delays in the access to the updated components of the iteration vector, is defined by

$$
r(p)=\left\{r_{1}(p), \ldots, r_{i}(p), \ldots, r_{\alpha}(p)\right\} \in N^{\alpha} \quad \text { for all } p \in N,
$$

where for all $i \in\{1, \ldots, \alpha\}$ and $p \in N, 0 \leq r_{i}(p) \leq p$ and $r_{i}(p)=0$ if $i=s(p)$.

We note that the latest condition has been introduced in order to simplify the analysis of the algorithms. This assumption is more restrictive than the one used in the classical model, however it is natural since it implies that each processor has access to its own working space without delay. In particular, this condition has been used in the framework of interval arithmetic (see [19]).

We introduce the functions $\rho_{i}: N \rightarrow N, i \in\{1, \ldots, \alpha\}$, defined by $\rho_{i}(p)=$ $p-r_{i}(p)$ and satisfying for all $i \in\{1, \ldots, \alpha\}$ and $p \in N$ :

$$
\begin{gathered}
0 \leq \rho_{i}(p) \leq p, \\
\rho_{i}(p)=p, \quad \text { if } i=s(p) .
\end{gathered}
$$

In the sequel, we will denote by $\rho(p)$ the mapping from $N^{\alpha}$ into itself, with components $\rho_{i}(p), i \in\{1, \ldots, \alpha\}$. We introduce now the sequences $\left\{K_{i}^{p}\right\}, p \in N$, defined by

$$
K_{i}^{p}=\{k \in N \mid s(k)=i, 0 \leq k<p\}, \quad i \in\{1, \ldots, \alpha\}, p \in N .
$$

The elements of the set $K_{i}^{p}$ correspond to the iteration numbers between 0 and $p$ at which the $i$ th block component of the iteration vector is updated. We note that

$$
\left\{K_{i}^{p}\right\} \text { is a denumerable sequence of finite elements of the set of parts of } N \text {. }
$$

Moreover $\left\{K_{i}^{p}\right\}$ is nested:

$$
K_{i}^{p} \subseteq K_{i}^{p+1} .
$$

Definition 8. Asynchronous iterations with order intervals associated with the $a$ supermapping $F^{a}$ are sequences $\left\{y^{p}\right\}$ defined recursively as follows. For all $p \in N$,

$$
\begin{cases}y_{i}^{p+1}=F_{i}^{a}\left(\tilde{y}^{p}\right), & \text { if } i=s(p), \\ y_{i}^{p+1}=y_{i}^{p}, & \text { if } i \neq s(p),\end{cases}
$$


where $\tilde{y}^{p}$ is implicitly defined by

$$
\tilde{y}^{0}=y^{0}, \text { an } a \text {-supersolution }
$$

and

$$
\tilde{y}^{p} \in\left\langle y^{p}, y^{\rho(p)} \wedge \tilde{y}^{q}\right\rangle, \quad \text { if } p \geq 1,
$$

where the block-components of the vector $y^{\rho(p)} \in E$ are the subvectors $y_{i}^{\rho_{i}(p)}, i \in$ $\{1, \ldots$,

$\alpha\}$, and $q=\max _{k \in K_{s(p)}^{p}} k$.

We note that $\tilde{y}^{p} \in\left\langle y^{p}, y^{\rho(p)}\right\rangle$ if $K_{i}^{p}=\varnothing, i=1, \ldots, \alpha$, where $\varnothing$ denotes the empty set.

Remark 4. Asynchronous iterations defined by (3.7)-(3.9) are general iterative methods whereby iterations are carried out in parallel by $\alpha$ processors in arbitrary order and possibly without any synchronization. Flexible communication between processors is the main feature of this class of algorithms. Indeed we note that the block components $\tilde{y}_{j}^{p}$ of the iteration vector used at the time of the updating of $y_{i}^{p+1}$ are taken anywhere in the order interval $\left\langle y_{j}^{p}, y_{j}^{\rho(p)} \wedge \tilde{y}_{j}^{q}\right\rangle_{j}$, where $\tilde{y}_{j}^{q}$ denotes the value used at the time of the last updating of the block-component $y_{i}$ and $y_{j}^{\rho(p)}$ is related to the non-deterministic behaviour of the iterative scheme. The introduction of order intervals permits us to take into account data coming from computations which are in progress and which are not explicitly labelled by an iteration number. So, there is a better coupling between communication and computation and we may expect a faster convergence. Moreover each communication of a block subvector is not necessarily associated with a given iteration number. The exchanged values of the components of each block subvector can be relative to different iteration numbers. We note that the model presented in this study permits us to analyse asynchronous block iterative methods directly in the block decomposition framework. So, it is not necessary to introduce a model defined with respect to individual components that would be artificial and cumbersome in the context of this study. The use of $a$-supermappings which approximate the solution of subproblems is another important feature of the parallel methods presented here.

Proposition 6. Let a be a continuous surjective $M$-function, $F$ the fixed point mapping defined by (2.7) and (2.8), $F^{a}$ an a-supermapping associated with $F$, $y^{0} \in E$ an a-supersolution, $\mathcal{S}$, and $\mathcal{R}$ a steering and a sequence of delays, respectively. Then the asynchronous iteration $\left\{y^{p}\right\}$ given by (3.7)-(3.9) is well defined and satisfies

$$
y^{*} \leq \cdots \leq y^{p+1} \leq y^{p} \leq \cdots \leq y^{0},
$$

and $\lim _{p \rightarrow \infty} y^{p}=y^{*}$, where $y^{*}$ is an a-supersolution of problem (2.5).

Proof. We proceed by induction. It follows from the equality (3.8) that

$$
a_{s(0)}\left(\tilde{y}^{0}\right)=a_{s(0)}\left(y^{0}\right) \geq 0 .
$$

Thus, the relations (3.7) and (3.10) imply the existence of a unique $y^{1} \in E$. By the definition of the $a$-supermapping $F^{a}$, it follows from (3.7) and (3.8) that

$$
y_{s(0)}^{1} \leq \tilde{y}_{s(0)}^{0}=y_{s(0)}^{0} \text {. }
$$


Moreover, it follows from (3.7) and (3.11) that

$$
y^{1} \leq y^{0} .
$$

It follows from the definition of the $a$-supermapping $F^{a},(3.7)$ and (3.8) that

$$
a_{i}\left(y^{1}\right)=a_{i}\left(y_{i}^{1} ; y^{0}\right) \geq 0, \quad \text { if } i=s(0) .
$$

Moreover, by the off-diagonal antitonicity of $a$ it follows from (3.7), (3.12), and (3.8) that

$$
a_{i}\left(y^{1}\right)=a_{i}\left(y_{i}^{0} ; y^{1}\right) \geq a_{i}\left(y^{0}\right) \geq 0, \quad \text { if } i \neq s(0) .
$$

The inequalities (3.13) and (3.14) imply

$$
a\left(y^{1}\right) \geq 0 .
$$

The relations (3.10), (3.12), and (3.15) give the first step of the induction. We assume now that for some $p$ there exists a unique $y^{m} \in E$, for all $m, 0<m \leq p$ and a unique $\tilde{y}^{m} \in E$, for all $m, 0 \leq m<p$, such that

$$
\begin{gathered}
a_{s(m)}\left(\tilde{y}^{m}\right) \geq 0, \\
y^{p} \leq \cdots \leq y^{m} \leq y^{m-1} \leq \cdots \leq y^{0}, \\
a\left(y^{m}\right) \geq 0 .
\end{gathered}
$$

It follows from (3.17), (3.3), and (3.9) that

$$
\tilde{y}_{s(m)}^{m}=y_{s(m)}^{m}, \quad \text { for all } m \leq p .
$$

From the relations (3.4) and (3.7) we have

$$
y_{s(p)}^{p}=y_{s(p)}^{0}, \quad \text { if } K_{s(p)}^{p}=\varnothing .
$$

It follows from the relations (3.17), (3.9), and (3.2) that

$$
\tilde{y}^{p} \leq y^{0}, \quad \text { if } K_{s(p)}^{p}=\varnothing .
$$

By the off-diagonal antitonicity of $a$ it follows from the relations (3.19)-(3.21) that

$$
a_{s(p)}\left(\tilde{y}^{p}\right)=a_{s(p)}\left(y_{s(p)}^{0} ; \tilde{y}^{p}\right) \geq a_{s(p)}\left(y^{0}\right) \geq 0, \quad \text { if } K_{s(p)}^{p}=\varnothing .
$$

Consider now the case where $K_{s(p)}^{p} \neq \varnothing$. From (3.7) and (3.4) we have

$$
y_{s(p)}^{p}=y_{s(q)}^{q+1}, \quad \text { if } K_{s(p)}^{p} \neq \varnothing,
$$

where

$$
q=\max _{k \in K_{s(p)}^{p}} k .
$$

Thus, from the equalities (3.19) and (3.23) we have

$$
\tilde{y}_{s(p)}^{p}=y_{s(p)}^{p}=y_{s(q)}^{q+1}, \quad \text { if } K_{s(p)}^{p} \neq \varnothing .
$$

Moreover, from the relation (3.9) we find that

$$
\tilde{y}^{p} \leq \tilde{y}^{q}, \quad \text { if } K_{s(p)}^{p} \neq \varnothing .
$$

By the off-diagonal antitonicity of $a$ and the definition of the $a$-supermapping $F^{a}$, it follows from the relations (3.25), (3.26), and (3.16) that

$$
a_{s(p)}\left(\tilde{y}^{p}\right)=a_{s(q)}\left(y_{s(q)}^{q+1} ; \tilde{y}^{p}\right) \geq a_{s(q)}\left(y_{s(q)}^{q+1} ; \tilde{y}^{q}\right) \geq 0, \quad \text { if } K_{s(p)}^{p} \neq \varnothing .
$$

We note that the relations (3.22) and (3.27) extend the inequality (3.16) to rank $p+1$. The relations (3.7)-(3.9), (3.22), and (3.27) imply the existence of a unique 
$y^{p+1}$. By the definition of the $a$-supermapping $F^{a}$, it follows from the relations (3.7) and (3.19) that

$$
y_{s(p)}^{p+1} \leq \tilde{y}_{s(p)}^{p}=y_{s(p)}^{p} .
$$

Thus, it follows from the relations (3.7) and (3.28) that

$$
y^{p+1} \leq y^{p} .
$$

We note that (3.29) extends the inequality (3.17) to rank $p+1$. By the off-diagonal antitonicity of $a$ and the definition of the $a$-supermapping $F^{a}$, it follows from the relations (3.29), (3.9), and (3.7) that

$$
a_{s(p)}\left(y^{p+1}\right) \geq a_{s(p)}\left(y_{s(p)}^{p+1} ; y^{p}\right) \geq a_{s(p)}\left(y_{s(p)}^{p+1} ; \tilde{y}^{p}\right) \geq 0 .
$$

Moreover, by the off-diagonal antitonicity of $a$ it follows from (3.7), (3.29), and (3.18) that

$$
a_{j}\left(y^{p+1}\right)=a_{j}\left(y_{j}^{p} ; y^{p+1}\right) \geq a_{j}\left(y^{p}\right) \geq 0, \quad \text { if } j \neq s(p) .
$$

The inequalities (3.30) and (3.31) imply

$$
a\left(y^{p+1}\right) \geq 0 .
$$

The inequality (3.32) extends (3.18) to rank $p+1$. Thus, the induction is complete. By the surjectivity of $a$, there exists $\bar{y} \in E$ such that

$$
a(\bar{y})=0 .
$$

Moreover, we have

$$
a\left(y^{p}\right) \geq 0, \quad \text { for all } p .
$$

By the inverse isotonicity of $a$ it follows from the relations (3.33) and (3.34) that $\bar{y} \leq y^{p}$, for all $p$.

Hence, there exists $y^{*}$ such that $\bar{y} \leq y^{*}, y^{*} \leq y^{p}$ for all $p$, and

$$
\lim _{p \rightarrow \infty} y^{p}=y^{*} .
$$

By the continuity of the mapping $a$ it follows from the relations (3.34) and (3.35) that

$$
a\left(y^{*}\right) \geq 0 .
$$

Thus, $y^{*}$ is an $a$-supersolution.

\section{TWO ClASSES OF $a$-SUPERMAPPINGS AND ASSOCIATED CONVERGENCE RESULTS}

\section{1. $a$-supermappings of the first kind.}

Definition 9. Let $F^{a}$ and $F^{b}$ be two a-supermappings associated with $F$ with components $F_{i}^{a}$ and $F_{i}^{b}$ respectively, defined on the domain $\left\{y \in E \mid a_{i}(y) \geq 0\right\}$. Then $F^{a}$ and $F^{b}$ satisfy the relation

$$
F^{a} \alpha F^{b},
$$

if for all $i \in\{1, \ldots, \alpha\}$ and $y$ element of the domain $\left\{y \in E \mid a_{i}(y) \geq 0\right\}$, we have

$$
F_{i}^{b}(y) \in\left\langle F_{i}^{a}(y), y_{i}\right\rangle_{i} .
$$


Definition 10. The mapping $F^{a}$ with components $F_{i}^{a}$ is an $a$-supermapping of the first kind associated with $F$ if $F^{a}$ is an $a$-supermapping associated with $F$, there exists an $a$-supermapping $F^{b}$ associated with $F$ such that $F^{a} \alpha F^{b}$, and

$$
y^{p} \downarrow y^{*}, p \rightarrow \infty \quad \text { implies } \quad F_{i}^{b}\left(y^{p}\right) \downarrow F_{i}^{b}\left(y^{*}\right), p \rightarrow \infty \text { for all } i \in\{1, \ldots, \alpha\},
$$

where the notation $y^{p} \downarrow y^{*}, p \rightarrow \infty$ means that

$$
y^{*} \leq \cdots \leq y^{p+1} \leq y^{p} \leq \cdots \leq y^{0} \quad \text { and } \quad \lim _{p \rightarrow \infty} y^{p}=y^{*} .
$$

Remark 5. The relation (4.2) can be interpreted as a property of continuity of $F^{b}$ related to the partial ordering.

Proposition 7. Let the assumptions of Proposition 6 hold. Let $F^{a}$ be an a-supermapping of the first kind associated with $F$ and assume that the steering $\mathcal{S}$ satisfies

$$
\begin{array}{r}
\text { for all } i \in\{1, \ldots, \alpha\} \text {, the set }\{p \in N \mid s(p)=i\} \text { is infinite and } \\
\text { for all } i \in\{1, \ldots, \alpha\}, \quad \lim _{p \rightarrow \infty} \rho_{i}(p)=+\infty .
\end{array}
$$

Then the sequence $\left\{y^{p}\right\}$ defined by (3.7)-(3.9) satisfies $y^{p} \downarrow \bar{y}$, where $\bar{y}$ is the unique solution of problem (2.5).

Proof. It follows from (3.3), (3.4), and (3.7) that for all $p \geq 1$ such that $s(p)=i$ and $K_{i}^{p} \neq \varnothing$, we have

$$
y_{i}^{\rho_{i}(p)}=y_{i}^{p}=\cdots=y_{i}^{q+1}=F_{i}^{a}\left(\tilde{y}^{q}\right),
$$

where

$$
q=\max _{k \in K_{s(p)}^{p}} k .
$$

We introduce now the sequence $\left\{z^{m}\right\}$ such that for all $i \in\{1, \ldots, \alpha\}$,

$$
\begin{gathered}
z_{i}^{2 m}=y_{i}^{p}, \quad m \geq 0, \\
z_{i}^{2 m-1}=F_{i}^{b}\left(\tilde{y}^{q}\right), \quad m \geq 1,
\end{gathered}
$$

where $m=\operatorname{card} K_{i}^{p}, s(p)=i$, and $q=\max _{k \in K_{i}^{p}} k$. It follows from Proposition 6 and (4.7) that

$$
z_{i}^{2 m} \downarrow y_{i}^{*}, m \rightarrow \infty \quad \text { for all } i \in\{1, \ldots, \alpha\},
$$

where $y^{*}$ is an $a$-supersolution. It follows from the relations (4.8), (4.1), (3.19), (4.5), and (4.7) that for all $i \in\{1, \ldots, \alpha\}$ and $m \geq 1$, we have

$$
z_{i}^{2 m-1}=F_{i}^{b}\left(\tilde{y}^{q}\right) \in\left\langle F_{i}^{a}\left(\tilde{y}^{q}\right), y_{i}^{q}\right\rangle_{i}=\left\langle z_{i}^{2 m}, z_{i}^{2 m-2}\right\rangle_{i} .
$$

Consider now the following notation. For all $i \in\{1, \ldots, \alpha\}$ and $l \in N$,

$$
z_{i}^{l}= \begin{cases}z_{i}^{2 m} & \text { if } l=2 m, \\ z_{i}^{2 m-1} & \text { if } l=2 m-1 .\end{cases}
$$

The relations (4.9)-(4.11) imply that

$$
z_{i}^{l} \downarrow y_{i}^{*}, l \rightarrow \infty, \quad \text { for all } i \in\{1, \ldots, \alpha\} .
$$

We note that $\lim _{p \rightarrow \infty} \operatorname{card} K_{i}^{p}=+\infty$ since

$$
K_{i}^{p}=\{k \in N \mid s(k)=i \text { and } 0 \leq k<p\} \subset\{k \in N \mid s(k)=i\},
$$


and the set $\{k \in N \mid s(k)=i\}$ is infinite from (4.3). Hence $q=\max _{k \in K_{i}^{p}} k \rightarrow+\infty$, if $m=\operatorname{card} K_{i}^{p} \rightarrow+\infty$. So, it follows from the relations (4.12) and (4.8) that for all $i \in\{1, \ldots, \alpha\}$,

$$
y_{i}^{*}=\lim _{m \rightarrow \infty} z_{i}^{2 m-1}=\lim _{q \rightarrow \infty} F_{i}^{b}\left(\tilde{y}^{q}\right) .
$$

It follows from (3.9) and (4.4) that

$$
\tilde{y}^{q} \downarrow y^{*}, \quad q \rightarrow \infty .
$$

Thus, the relations (4.13), (4.14), and (4.2) imply

$$
y_{i}^{*}=\lim _{q \rightarrow \infty} F_{i}^{b}\left(\tilde{y}^{q}\right)=F_{i}^{b}\left(y^{*}\right), \quad \text { for all } i \in\{1, \ldots, \alpha\} .
$$

It follows from Definition 4 that $F_{i}^{b}\left(y^{*}\right)=y_{i}^{*}$ implies $F_{i}\left(y^{*}\right)=y_{i}^{*}$, for all $i \in$ $\{1, \ldots, \alpha\}$. Hence, $y^{*}=\bar{y}$ which is the unique solution of problem $(2.5)$.

\section{2. $a$-supermappings of the second kind.}

Definition 11. The mapping $F^{a}$ with components $F_{i}^{a}$ is an $a$-supermapping of the second kind associated with $F$ if $F^{a}$ is an $a$-supermapping associated with $F$ and for all $i \in\{1, \ldots, \alpha\}$ and $y$ element of the domain of definition of $F_{i}^{a}:\{y \in E \mid$ $\left.a_{i}(y) \geq 0\right\}$, there exists $\delta>0$ such that

$$
\left\|y_{i}-F_{i}^{a}(y)\right\|_{i} \geq \delta\left\|y_{i}-F_{i}(y)\right\|_{i},
$$

where $\|\cdot\|_{i}$ is a norm defined in $E_{i}$.

Proposition 8. Let the assumptions of Proposition 7 hold and assume that $F^{a}$ is an a-supermapping of the second kind associated with $F$. Then the sequence $\left\{y^{p}\right\}$ defined by (3.7)-(3.9) satisfies $y^{p} \downarrow \bar{y}$, where $\bar{y}$ is the unique solution of problem $(2.5)$.

Proof. It follows from Proposition 6 that $y^{p} \downarrow y^{*}$. Then for all $i \in\{1, \ldots, \alpha\}$ and $\varepsilon>0$, there exists $p(\varepsilon) \in N$, such that for all $p \geq p(\varepsilon)$ with $s(p)=i$, we have

$$
\left\|y_{i}^{p}-y_{i}^{*}\right\|_{i} \leq \frac{\varepsilon}{2} . \delta \quad \text { and } \quad\left\|y_{i}^{p+1}-y_{i}^{*}\right\|_{i} \leq \frac{\varepsilon}{2} . \delta .
$$

Then

$$
\left\|y_{i}^{p}-y_{i}^{p+1}\right\|_{i} \leq \varepsilon . \delta .
$$

It follows from (4.16), (3.7), (3.3), (3.9), and (4.15) that for all $i \in\{1, \ldots, \alpha\}$ and $\varepsilon>0$, there exists $p(\varepsilon) \in N$, such that for all $p \geq p(\varepsilon)$ with $s(p)=i$, we have

$$
\left\|\tilde{y}_{i}^{p}-F_{i}\left(\tilde{y}^{p}\right)\right\|_{i} \leq \varepsilon
$$

It follows from Proposition 6, (3.9), and (4.4) that

$$
\tilde{y}^{p} \downarrow y^{*}, \quad p \rightarrow \infty .
$$

Let $i=s(p)$. We now introduce the following notation: $u^{p}(i)=\left\{\ldots, u_{j}^{p}(i), \ldots\right\}$, where

$$
u_{j}^{p}(i)= \begin{cases}\tilde{y}_{j}^{p} & \text { if } j \neq i \\ F_{i}\left(\tilde{y}^{p}\right) & \text { if } j=i\end{cases}
$$


From (2.7) and (2.8) we find that

$$
a_{i}\left(u^{p}(i)\right)=0, \quad \text { for all } p \in N \text { and } i \in\{1, \ldots, \alpha\} \text { such that } i=s(p) .
$$

It follows from (4.17) and (4.18) that for all $i \in\{1, \ldots, \alpha\}$, we have

$$
\lim _{p \rightarrow \infty} u^{p}(i)=y^{*} \text {. }
$$

By the continuity of $a$ it follows from (4.19) and (4.20) that

$$
a_{i}\left(y^{*}\right)=0, \quad \text { for all } i \in\{1, \ldots, \alpha\} .
$$

\section{Application to a Class of nOnlinear simultaneous EQUATions}

We consider in this section an $M$-function obtained by a diagonal monotone perturbation of an $M$-matrix $A$

$$
a(x)=A x-b+\varphi(x),
$$

where $b \in R^{n}, \varphi(x)=\operatorname{diag}\left\{\ldots, \varphi_{i}\left(x_{i}\right), \ldots\right\}$, and the point-to-point functions $\varphi_{i}$ are monotone increasing. Moreover, the functions $\varphi_{i}$ are also continuous. Consider now the solution of the following nonlinear simultaneous equations

$$
a(x)=0,
$$

by asynchronous iterations with order intervals derived from the Schwarz alternating subdomain method.

Remark 6. The results of this section can be extended to the case where $\varphi(x)$ is a diagonal monotone maximal and possibly multivalued operator (see [1]). Then we must solve the following nonlinear algebraic problem

$$
b-A x \in \varphi(x) .
$$

5.1. Connection with the discrete Schwarz alternating procedure. To simplify the presentation we consider in this subsection the case of two discrete subdomains that may overlap. We introduce $m_{1}, m_{2}$ and $n$ which are three integers, $n$ is the number of discretized points, $m_{1}$ denotes the last index of the first subdomain, and $m_{2}$ the first index of the second subdomain, then

$$
1<m_{2}<m_{1}<n .
$$

We introduce also the sets

$$
\mathcal{T}_{1}=\left\{1, \ldots, m_{1}\right\}, \quad \mathcal{T}_{2}=\left\{m_{2}, \ldots, n\right\}, \quad \mathcal{T}^{\prime}=\mathcal{T}_{1} \cap \mathcal{T}_{2}=\left\{m_{2}, \ldots, m_{1}\right\} .
$$

Let $\delta m=m_{1}-m_{2}+1$ and $m=n+\delta m$. We will consider in the sequel augmented vectors of $R^{m}$ and use the following notations:

$$
\begin{gathered}
\widetilde{\mathcal{T}}_{1}=\left\{1, \ldots, m_{1}\right\}, \quad \widetilde{\mathcal{T}}_{2}=\left\{m_{1}+1, \ldots, m\right\}, \\
\widetilde{\mathcal{T}}_{1}^{\prime}=\left\{m_{2}, \ldots, m_{1}\right\}, \quad \widetilde{\mathcal{T}}_{2}^{\prime}=\left\{m_{1}+1, \ldots, m_{1}+\delta m\right\},
\end{gathered}
$$

where $\widetilde{\mathcal{T}}_{1}^{\prime}$ and $\widetilde{\mathcal{T}}_{2}^{\prime}$ correspond to the overlapping. We define the mappings $\tilde{p}^{1}, \tilde{p}^{2}: R^{n}$ $\rightarrow R^{m}$ as follows. For all $u \in R^{n}$, the mappings $\tilde{p}^{1}(u), \tilde{p}^{2}(u)$, with components 
$\tilde{p}_{l}^{1}(u), \tilde{p}_{l}^{2}(u)$, respectively, are given by

$$
\begin{aligned}
& \tilde{p}_{l}^{1}(u)= \begin{cases}u_{l} & \forall l \in \widetilde{\mathcal{T}}_{1}, \\
0, & \forall l \in \widetilde{\mathcal{T}}_{2}^{\prime}, \\
u_{l-\delta m}, & \forall l \in \widetilde{\mathcal{T}}_{2} \ominus \widetilde{\mathcal{T}}_{2}^{\prime},\end{cases} \\
& \tilde{p}_{l}^{2}(u)= \begin{cases}u_{l}, & \forall l \in \widetilde{\mathcal{T}}_{1} \ominus \widetilde{\mathcal{T}}_{1}^{\prime}, \\
0, & \forall l \in \widetilde{\mathcal{T}}_{1}^{\prime}, \\
u_{l-\delta m}, & \forall l \in \widetilde{\mathcal{T}}_{2} .\end{cases}
\end{aligned}
$$

We define also the mappings $r^{1}, r^{2}: R^{m} \rightarrow R^{n}$ as follows: for all $\tilde{u} \in R^{m}$, the mappings $r^{1}(\tilde{u}), r^{2}(\tilde{u})$ with components $r_{l}^{1}(\tilde{u}), r_{l}^{2}(\tilde{u})$, respectively, are given by

$$
\begin{aligned}
& r_{l}^{1}(\tilde{u})= \begin{cases}\tilde{u}_{l}, & \forall l \in \mathcal{T}_{1}, \\
\tilde{u}_{l+\delta m}, & \forall l \in \mathcal{T}_{2} \ominus \mathcal{T}^{\prime},\end{cases} \\
& r_{l}^{2}(\tilde{u})= \begin{cases}\tilde{u}_{l}, & \forall l \in \mathcal{T}_{1} \ominus \mathcal{T}^{\prime}, \\
\tilde{u}_{l+\delta m}, & \forall l \in \mathcal{T}_{2} .\end{cases}
\end{aligned}
$$

Remark 7. For all $i \in\{1,2\}$ and $u \in R^{n}$, we have $r^{i}\left(\tilde{p}^{i}(u)\right)=u$. The mappings $\tilde{p}^{1}, \tilde{p}^{2}\left(r^{1}, r^{2}\right)$ transform a vector of size $n(m)$ into a vector of size $m(n)$.

We consider now the following system of equations

$$
\tilde{a}(\tilde{u})=0,
$$

where the mapping $\tilde{a}$ from $R^{m}$ into $R^{m}$ with components $\tilde{a}_{k}(\tilde{u}), k=1, \ldots, m$, is defined as follows:

$$
\begin{cases}\tilde{a}_{k}(\tilde{u})=a_{k}\left(r^{1}(\tilde{u})\right), & \forall k \in \widetilde{\mathcal{T}}_{1}, \\ \tilde{a}_{k}(\tilde{u})=a_{k-\delta m}\left(r^{2}(\tilde{u})\right), & \forall k \in \widetilde{\mathcal{T}}_{2} .\end{cases}
$$

Proposition 9 (see [17]). Let $A \in \mathcal{L}\left(R^{n} ; R^{n}\right)$ be an $M$-matrix. Then the matrix $\widetilde{A} \in \mathcal{L}\left(R^{m} ; R^{m}\right)$ which results from the above augmentation process is also an $M-$ matrix.

Remark 8. The nonlinear diagonal operator $\varphi$ from $R^{n}$ onto itself can be extended similarly.

Corollary 1. Under the assumptions of this section, the mapping $\tilde{a}$ defined by (5.5) is a surjective $M$-function.

Remark 9. The cases with $\alpha$ subdomains $(\alpha>2)$ where no components belong to more than two subdomains can be reduced to the case where $\alpha=2$ by considering a banded subdomain decomposition with red-black ordering.

\subsection{Application to the solution of nonlinear partial differential equa-}

tions. An illustration of the above theoretical study is given in this subsection. We consider two examples.

Example 1. Nonlinear diffusion problem. We shall primarily be concerned with the following problem.

Find $u$ such that

$$
\left\{\begin{array}{l}
-\Delta u+a \frac{\partial u}{\partial x}+b \frac{\partial u}{\partial y}+e^{c u}=f \quad \text { everywhere in } \Omega \\
u / \partial \Omega=0
\end{array}\right.
$$


where $\Omega$ is an open domain of $R^{2}$ (or $R^{3}$ ), $\partial \Omega$ is the boundary of $\Omega, a, b, c$ are real numbers, and $c$ is positive. We consider the discretization of the problem (5.6) using five-point difference equations for the Laplace operator and only one-sided backward or forward difference equations, according to the sign of $a$ and $b$, for the first derivatives of $u$. Then we have the system

$$
\sum_{j=1}^{\alpha} A_{i j} x_{j}+\varphi_{i}\left(x_{i}\right)=b_{i}, \quad \text { for all } i \in\{1, \ldots, \alpha\},
$$

where $x_{i}, b_{i} \in E_{i}$, and

$$
\varphi_{i}\left(x_{i}\right) \text { is isotone, convex, and continuously differentiable. }
$$

We note that the matrix $A$ is block-partitioned. Moreover it follows from the above discretization that $A$ is an $M$-matrix.

Remark 10. We can obtain a discretized system with the same properties using suitable $P_{1}$ finite element methods.

The problem (5.7) is similar to (2.5). Let $a_{i}\left(z_{i} ; w\right)=A_{i i} z_{i}+\varphi_{i}\left(z_{i}\right)+\sum_{j \neq i} A_{i j} w_{j}-$ $b_{i}$. We can define implicitly a fixed point mapping $F$ from $R^{n}$ into $R^{n}$ satisfying (2.8) and associated with problem (5.7).

Let $w, x^{0}$, and $z^{0}$ be three vectors of $R^{n}$ such that

$$
a_{i}(w) \geq 0, z_{i}^{0}=w_{i}, \quad \text { and } \quad x_{i}^{0}=z_{i}=F_{i}(w), \quad \text { for all } i \in\{1, \ldots, \alpha\} .
$$

Under the above assumptions the mapping $a_{i}$ is an $M$-function on the order interval $\left\langle x_{i}^{0}, z_{i}^{0}\right\rangle_{i}$.

We introduce the block-diagonal matrix $C(w)$, derived from Newton's method, with diagonal blocks $C_{i}(w)$ given by

$$
C_{i}(w)=A_{i i}+\varphi_{i}^{\prime}(w) .
$$

The matrix $C_{i}(w)$ is an $M$-matrix since $\varphi_{i}$ is convex and the matrix $A_{i i}$ is an $M$-matrix. Consider now the mappings $F^{a}$ with components $F_{i}^{a}$ defined by

$$
F_{i}^{a}(w)=z_{i}^{k}, \quad \text { for all } i \in\{1, \ldots, \alpha\},
$$

where $k$ is an integer, $z_{i}^{0}=w_{i}$, and $z_{i}^{k}$ is the $k$ th iteration of the following algorithm:

$$
z^{k+1}=z^{k}-C^{-1}\left(z^{\rho^{\prime}(k)}\right) \cdot a\left(z^{k}\right), \quad k=0,1, \ldots,
$$

where $0 \leq \rho^{\prime}(k) \leq k$. We first consider the mapping $F^{b}$ with components $F_{i}^{b}$ given by $F_{i}^{b}(w)=z_{i}^{1}$, for all $i \in\{1, \ldots, \alpha\}$.

Proposition 10. The mapping $F^{b}$ is an a-supermapping associated with $F$.

Proof. It follows from the definition of $z^{0}$ that $a\left(z^{0}\right) \geq 0$. Moreover $C^{-1}\left(z^{0}\right) \geq 0$ since $C\left(z^{0}\right)$ is an $M$-matrix. Then

$$
z^{1}-z^{0}=-C^{-1}\left(z^{0}\right) a\left(z^{0}\right) \leq 0 .
$$

It follows from the convexity of $\varphi$ (see [31, p. 448]) that

$$
a\left(z^{0}\right)-a(\bar{z}) \leq C\left(z^{0}\right)\left(z^{0}-\bar{z}\right) .
$$

Thus if $\bar{z}$ is the solution of the problem $a(z)=0$, then

$$
\bar{z}=\bar{z}-C^{-1}\left(z^{0}\right) a(\bar{z})=z^{1}-\left(z^{0}-\bar{z}\right)+C^{-1}\left(z^{0}\right)\left(a\left(z^{0}\right)-a(\bar{z})\right)
$$


and

$$
z^{1}-\left(z^{0}-\bar{z}\right)+C^{-1}\left(z^{0}\right)\left(a\left(z^{0}\right)-a(\bar{z})\right) \leq z^{1}-\left(I-C^{-1}\left(z^{0}\right) \cdot C\left(z^{0}\right)\right)\left(z^{0}-\bar{z}\right)=z^{1} .
$$

Thus

$$
\bar{z} \leq z^{1}
$$

Moreover it follows from the convexity of $\varphi$ that

$$
0=a\left(z^{0}\right)+C\left(z^{0}\right)\left(z^{1}-z^{0}\right) \leq a\left(z^{1}\right) .
$$

We can show analogously by induction (see [37]) that

$$
\bar{z} \leq \cdots \leq z^{k} \leq z^{k-1} \leq \cdots \leq z^{0} \text { and } a\left(z^{k}\right) \geq 0 .
$$

Thus the mappings $F^{a}$ are $a$-supermappings associated with $F$. Moreover it follows from (5.10), (5.12), and Definition 9 that

$$
F^{a} \alpha F^{b} \text {. }
$$

We recall that $F_{i}^{b}(w)=z_{i}^{1}=w-C^{-1}(w) \cdot a(w)$, where $C(w)$ is a block-diagonal matrix with blocks $C_{i}(w)=A_{i i}+\varphi_{i}^{\prime}(w)$. It follows from (5.8) that the mapping $a(w)$ given by

$$
a(w)=A w+\varphi(w)-b,
$$

is continuous. Moreover we have

$$
\left(A_{i i}+\varphi_{i}^{\prime}\left(w_{i}\right)\right)^{-1} \leq A_{i i}^{-1}
$$

since $A$ is an $M$-matrix and $\varphi^{\prime}$ is positive (see [31, p. 55]). It follows that the spectral radius of $C^{-1}(w)$ is less than or equal to the spectral radius of the inverse of the block-diagonal matrix with blocks $A_{i i}$ which is an $M$-matrix. Thus the linear application associated with the matrix $C^{-1}(w)$ is Lipschitz continuous and $C^{-1}(w)$ is uniformly Lipschitz continuous. By the continuity of $a(w), C(w)$ and the fact that $C^{-1}(w)$ is uniformly Lipschitz continuous, we find that the mapping $F^{b}$ is continuous. So, the mappings $F^{a}$ defined by (5.9) are $a$-supermappings of the first kind associated with $F$.

Remark 11. Taking into account the norm convergence properties of Newton's method quoted in subsection 13.3.4 of [31], we can show that the above $a$-supermappings $F^{a}$ and $F^{b}$ are also $a$-supermappings of the second kind associated with $F$.

Example 2. The discretized and linearized Hamilton-Jacobi-Bellman problem. We consider the following problem

$$
\left\{\begin{array}{l}
\text { Find } u \text { such that } \\
\max \left\{\mathbf{A}^{1} u-f^{1}, \mathbf{A}^{2} u-f^{2}\right\}=0, \text { everywhere in } \Omega, \\
u / \partial \Omega=0
\end{array}\right.
$$

where $\mathbf{A}_{1}$ and $\mathbf{A}_{2}$ are two elliptic operators of second order satisfying the Maximum Principle and $f_{1}, f_{2}$ are elements of $L^{2}(\Omega)$.

Under appropriate discretization of problem (5.13) by finite differences and assuming in particular that the incidence matrices $B^{1}$ and $B^{2}$ associated with the discretization matrices are equal, we obtain the following discretized problem

$$
\left\{\begin{array}{l}
\text { Find } x \text { solution of } \\
\max \left(A^{1} \cdot x-b^{1}, A^{2} \cdot x-b^{2}\right)=0
\end{array}\right.
$$


where $b^{1}, b^{2} \in R^{n}$ and $A^{1}, A^{2}$ are matrices of size $n \times n$ with entries $a_{i j}^{1}, a_{i j}^{2}$, respectively, which satisfy

$$
\begin{gathered}
a_{i i}^{r}>0, \quad a_{i j}^{r} \leq 0, \quad i=1, \ldots, n, j=1, \ldots, n, j \neq i, r=1,2, \\
\sum_{j} a_{i j}^{r} \geq 0, \quad i=1, \ldots, n, r=1,2,
\end{gathered}
$$$$
\text { there exists at least one } i \text { such that } \sum_{j} a_{i j}^{1}>0 \text { and } \sum_{j} a_{i j}^{2}>0 \text {, }
$$

the matrices $A^{1}$ and $A^{2}$ are irreducible.

Remark 12. We recall that the incidence matrix $B^{1}\left(B^{2}\right)$ of size $n \times n$ is generated from the matrix $A^{1}\left(A^{2}\right)$ by replacing the entries $a_{i j}^{1}\left(a_{i j}^{2}\right)$ by 1 if $a_{i j}^{1} \neq 0\left(a_{i j}^{2} \neq 0\right)$.

We note that the matrices $A^{1}$ and $A^{2}$ are diagonally dominant. Under the above assumptions $A^{1}$ and $A^{2}$ are also $M$-matrices (see [38]). The problem (5.14) can be linearized as follows:

$$
a(x)=C(x) \cdot x-b(x)=0,
$$

where $b(x) \in R^{n}$ and $C(x)$ is a matrix of size $n \times n$. If $\left(A_{1} x-b_{1}\right)_{i}$ is greater than $\left(A_{2} x-b_{2}\right)_{i}$, then the $i$ th row of matrix $C(x)$ is equal to the $i$ th row of matrix $A_{1}$ else it is equal to the $i$ th row of matrix $A_{2}$. The vector $b(x)$ is defined analogously. It follows from the above assumptions that the matrix $C(x)$ is an irreducible diagonally dominant matrix; moreover $C(x)$ is an $M$-matrix (see [38]). Thus $a$ is an $M$-function.

The mapping $a_{i}\left(z_{i} ; w\right)$ defined by

$$
a_{i}\left(z_{i} ; w\right)=\max \left(A_{i i}^{1} z_{i}-\left(b_{i}^{1}-\sum_{j \neq i} A_{i j}^{1} w_{j}\right), A_{i i}^{2} z_{i}-\left(b_{i}^{2}-\sum_{j \neq i} A_{i j}^{2} w_{j}\right)\right)=0
$$

is a continuous surjective $M$-function since $C(x)$ is an $M$-matrix. We can define a fixed point mapping $F$ from $R^{n}$ into $R^{n}$ satisfying (2.8) and associated with problem (5.19).

Let $w, x^{0}, z^{0}$ be three vectors of $R^{n}$ such that

$$
a_{i}(w)=\max \left(\sum_{j} A_{i j}^{1} w_{j}-b_{i}^{1}, \sum_{j} A_{i j}^{2} w_{j}-b_{i}^{2}\right) \geq 0,
$$

$z_{i}^{0}=w_{i}$, and $x_{i}^{0}=z_{i}=F_{i}(w)$, for all $i \in\{1, \ldots, \alpha\}$. Let $x, z$ be two vectors of $R^{n}$ such that $x_{i}, z_{i} \in\left\langle x^{0}, z^{0}\right\rangle_{i}$, for all $i \in\{1, \ldots, \alpha\}$. Consider the following notation: for all $i \in\{1, \ldots, \alpha\}$,

$$
\begin{aligned}
& a_{i}(x)=\max _{t \in\{1,2\}}\left(A^{t} x-b^{t}\right)_{i}=\left(A^{r} x-b^{r}\right)_{i}, \\
& a_{i}(z)=\max _{t \in\{1,2\}}\left(A^{t} z-b^{t}\right)_{i}=\left(A^{s} z-b^{s}\right)_{i} .
\end{aligned}
$$

We have for all $i \in\{1, \ldots, \alpha\},\left(A^{s} x-b^{s}\right)_{i} \leq a_{i}(x)$. Then

$$
\left(A^{s} x-b^{s}\right)_{i}-\left(A^{r} x-b^{r}\right)_{i} \leq 0 .
$$

Hence we have

$$
\left(A^{s} z-b^{s}\right)_{i}-\left(A^{r} x-b^{r}\right)_{i}-\left(A^{s}(z-x)\right)_{i} \leq 0 .
$$


Thus for all $i \in\{1, \ldots, \alpha\}$,

$$
a_{i}(z)-a_{i}(x) \leq(C(z)(z-x))_{i},
$$

since the $i$ th line of $C(z)$ is the $i$ th line of $A^{s}(z)$. So the mapping $a$ is order-convex. We now define the mapping $F^{a}$ with components $F_{i}^{a}$ as follows:

$$
F_{i}^{a}(w)=z_{i}^{k}, \quad \text { for all } i \in\{1, \ldots, \alpha\},
$$

where $k$ is an integer and the vector $z^{k}$ is given by the following algorithm:

$$
z^{k+1}=z^{k}-C^{-1}\left(z^{\rho(k)}\right) a\left(z^{k}\right),
$$

which is derived from the fixed point equation $z=z-C^{-1}(z) a(z)$. We analogously define the mapping $F^{b}$ with components $F_{i}^{b}$ as follows:

$$
F_{i}^{b}(w)=z_{i}^{1}, \quad \text { for all } i \in\{1, \ldots, \alpha\} .
$$

By using an argument similar to the one developed in the proof of Proposition 10 it follows from (5.22) that the mappings $F^{a}$ and $F^{b}$ are two $a$-supermappings associated with $F$ which satisfy

$$
F^{a} \alpha F^{b}
$$

The continuity of the mapping $F^{b}$ follows from the continuity of the mapping $a$ since the convex hull of continuous functions of $R^{n}$ is continuous. Thus the mapping $F^{a}$ is an $a$-supermapping of the first kind associated with $F$.

Remark 13. The reader is referred to [23] for the solution of the Hamilton-JacobiBellman problem via several sequential algorithms. The first two algorithms proposed in [23] when used for the solution of the subproblems presented above can lead to $a$-supermappings of the first kind; in particular the second algorithm corresponds exactly to the linearization method (5.19). Note also that the methods presented in [23] are not related to relaxation or subdomain methods.

Remark 14. The obstacle stationary problem associated to a second order elliptic operator satisfying the maximum principle can classically be written as a HamiltonJacobi-Bellman problem, moreover the algorithms presented in this paper can be used for the numerical solution of this problem. Nevertheless the above study cannot be applied to this case since assumption (5.18) is not satisfied. The obstacle problem is important, but we have restricted attention to the case treated in Example 2 in order to a give a short presentation. The reader is referred to [37] for a detailed study of the obstacle stationary problem.

5.3. Numerical experiments. In this subsection we briefly present computational experience using a shared memory multiprocessor IBM 3090 with up to six vector processors.

We have considered the two problems presented in subsection 5.2 (i.e. the nonlinear diffusion problem and the discretized Hamilton-Jacobi-Bellman equations). The Schwarz alternating procedure with overlapping has been used. The efficiency of parallel iterative algorithms is reported in Table 1 using the classical definition of efficiency: $e=\frac{t_{1}}{t_{p}} \cdot \frac{1}{p}$, where $t_{p}$ denotes the computing time using $p$ processors. Results are given for discretized domains with 25000 points. From Table 1 it can be seen that the efficiency of asynchronous iterations with order intervals is better than the efficiency of parallel synchronous iterations. Idle time due to synchronization and overheads of synchronization reduce the efficiency of parallel iterative 
methods. Moreover the efficiency decreases as the number of processors increases. Details of the implementation and other computational results can be found in [37].

TABLE 1. Efficiency of parallel algorithms

\begin{tabular}{|c|c|c|c|c|}
\hline & \multicolumn{2}{|c|}{ nonlinear diffusion problem } & \multicolumn{2}{c|}{ Hamilton-Jacobi-Bellman problem } \\
\hline $\begin{array}{c}\text { number of } \\
\text { processors }\end{array}$ & $\begin{array}{c}\text { synchronous } \\
\text { algorithms }\end{array}$ & $\begin{array}{c}\text { asynchronous } \\
\text { algorithms }\end{array}$ & $\begin{array}{c}\text { synchronous } \\
\text { algorithms }\end{array}$ & $\begin{array}{c}\text { asynchronous } \\
\text { algorithms }\end{array}$ \\
\hline 3 & 0.87 & 0.99 & 0.86 & 0.90 \\
\hline 6 & 0.65 & 0.81 & 0.85 & 0.90 \\
\hline
\end{tabular}

\section{ACKNOWLEDGMENTS}

The authors wish to thank the referee for helpful criticism and improvement of their manuscript.

\section{REFERENCES}

[1] M. Bahi, and J.-C. Miellou, Contractive mappings with maximum norms: comparisons of constants of contraction and application to asynchronous iterations, Parallel Computing 19 (1993), 511-523. MR 94c:65072

[2] R. H. Barlow and D. J. Evans, Synchronous and asynchronous iterative parallel algorithms for linear systems, Comput. J. 25 (1982), 56-60.

[3] G. M. Baudet, Asynchronous iterative methods for multiprocessors, J. Assoc. Comput. Mach. 25 (1978), 226-244. MR 58:13674

[4] D. P. Bertsekas, Distributed dynamic programming, IEEE Trans. Auto. Contr. 27 (1982), 610-616. MR 83k:90124

[5] D. P. Bertsekas, Distributed asynchronous computation of fixed points, Mathematics Programming 27 (1983), 107-120. MR 85a:68051

[6] D. P. Bertsekas and D. El Baz, Distributed asynchronous relaxation methods for convex network flow problems, SIAM J. Control Optim. 25 (1987), 74-85. MR 88f:90061

[7] D. P. Bertsekas and J. Tsitsiklis, Parallel and distributed computation, Numerical Methods, Englewood Cliffs, Prentice Hall, 1989.

[8] D. Chazan and W. Miranker, Chaotic relaxation, Linear Algebra and Appl. 2 (1969), 199-222. MR 40:5114

[9] P. Cousot, Méthodes itératives de construction et d'approximation de points fixes d'opérateurs monotones sur un treillis, analyse sémantique des programmes, Thèse d'Etat, Université de Grenoble (1978).

[10] D. El Baz, M-functions and parallel asynchronous algorithms, SIAM J. Numer. Anal. 27 (1990), 136-140. MR 91e:65165

[11] D. El Baz, Asynchronous gradient algorithms for a class of convex separable network flow problems, Computational Optimization and Applications 5 (1996), 187-205. CMP 96:12

[12] D. El Baz, Asynchronous implementation of relaxation and gradient algorithms for convex network flow problems, Parallel Computing 19 (1993), 1019-1028.

[13] D. El Baz, Nonlinear systems of equations and parallel asynchronous iterative algorithms, Advances in Parallel Computing 9, North-Holland, Amsterdam, 1994, 89-96.

[14] D. El Baz, P. Spiteri, and J.-C. Miellou, Distributed asynchronous iterative methods with order intervals for a class of nonlinear optimization problems, Journal of Parallel and Distributed Computing 38 (1996), 1-15.

[15] M. N. El Tarazi, Some convergence results for asynchronous algorithms, Numerical Math. 39 (1982), 325-340. MR 84a:65041

[16] M. N. El Tarazi, Algorithmes mixtes asynchrones, Etude de la convergence monotone, Numer. Math. 44 (1984), 363-369. MR 86c:65042

[17] D. J. Evans and W. Deren, An asynchronous parallel algorithm for solving a class of nonlinear simultaneous equations, Parallel Computing 17 (1991), 165-180. MR 92f:65166 
[18] A. Frommer, On asynchronous iterations in partially ordered spaces, Numer. Funct. Anal. and Optimiz. 12 (1991), 315-325. MR 92m:54060

[19] A. Frommer and H. Schwandt, Asynchronous parallel methods for enclosing solutions of nonlinear equations, J. Comput. Appl. Math. 60 (1995), 47-62. MR 96f:65062

[20] L. Giraud and P. Spiteri, Résolution parallèle de problèmes aux limites non linéaires, RAIRO Modél. Math. Anal. Numér. 25 (1991), 73-100. MR 92d:65244

[21] L. Giraud and P. Spiteri, Implementations of parallel solutions for nonlinear boundary value problems, Parallel Computing '91, Advances in Parallel Computing (Evans, Joubert, Liddel, Editors), North-Holland, Amsterdam, 1992, 203-211. CMP 93:04

[22] R. W. Hockney and C. R. Jesshope, Parallel Computers 2, Adam Hilger, Bristol, 1988.

[23] P. L. Lions and B. Mercier, Approximation numérique des équations de Hamilton-JacobiBellman, RAIRO Analyse numérique 14 (1980), 369-393. MR 82b:65055

[24] J.-C. Miellou, Itérations chaotiques à retards, C.R. Acad. Sci. Paris 278 (1974), 957-960. MR 50:15325

[25] J.-C. Miellou, Itérations chaotiques à retards, étude de la convergence dans le cas d'espaces partiellement ordonnés, C. R. Acad. Sci. Paris 280 (1975), 233-236. MR 52:12320

[26] J.-C. Miellou, Algorithmes de relaxation chaotique à retards, RAIRO R1, (1975), 55-82. MR 55: 13772

[27] J.-C. Miellou, Asynchronous iterations and order intervals, Parallel Algorithms (M. Cosnard et al., eds.) North-Holland, Amsterdam, 1986, 85-96. MR 88f:65083

[28] J.-C. Miellou, Ph. Cortey-Dumont, and M. Boulbrachêne, Perturbation of fixed-point iterative methods, Advances in Parallel Computing 1, JAI Press Inc., 1990, 81-122.

[29] J.-C. Miellou and P. Spiteri, Un critère de convergence pour des méthodes générales de point fixe, RAIRO Modél. Math. Anal. Numér. (1985), 170-201. MR 87f:47098

[30] J. M. Ortega, Introduction to Parallel and Vector Solution of Linear Systems, Plenum Press, New York, 1988. MR 92i:65220a

[31] J. M. Ortega and W. C. Rheinboldt, Iterative Solutions of Nonlinear Equations in Several Variables, Academic Press, New York, 1970. MR 42:8686

[32] W. C. Rheinboldt, On M-functions and their application to nonlinear Gauss-Seidel iterations and to network flows, J. Math. Anal. and Appl. 32 (1970), 274-307. MR 42:3997

[33] F. Robert, M. Charnay, and F. Musy, Itérations chaotiques série parallèle pour des équations non linéaires de point fixe, Aplikace Math. 20 (1975), 1-38. MR 51:9472

[34] U. Schendel, Introduction to Numerical Methods for Parallel Computers, Ellis Horword, Chichester, 1984. MR 87j:65003

[35] P. Spiteri, Simulation d'exécutions parallèles pour la résolution d'inéquations varitionnelles stationnaires, 1er Coll. Int. GAMNI Méthodes Vectorielles et Parallèles en Calcul Scientifique, Paris, Rev. EDF Ser. C (1) (1983), 149-159. MR 85b:65122

[36] P. Spiteri, Parallel asynchronous algorithms for solving boundary value problems, in Parallel Algorithms (M. Cosnard et al., eds.), North-Holland, Amsterdam, 1986, 73-84. MR 88a:65158

[37] P. Spiteri, J.-C. Miellou and D. El Baz, Asynchronous alternating Schwarz method for the solution of nonlinear partial differential equations, IRIT report 95-17-R, LCS 195.10, LAAS Report 95309 (1995).

[38] R. Varga, Matrix iterative analysis, Prentice Hall, Englewood Cliffs, 1962. MR 28:1725

L.C.S. URA CNRS Nº 040741, Université de Franche-Comté, 16, Route de Gray, 25030 Besançon Cedex, France

E-mail address: miellou@comte.univ-fcomte.fr

LAAS Du CNRS L.P. CNRS 8001, 7, Avenue du Colonel Roche, 31077 Toulouse Cedex, FRANCE

ENSEeiht-IRIT UA CNRS 1399, Lima, Institut National Polytechnique de Toulouse, 2, Rue Camichel, 31071 Toulouse Cedex, France 